\title{
Procesos de socialización y de acumulación de capitales social y cultural en estudiantes innovadores de educación primaria y secundaria en Costa Rica
}

\author{
Alexis Segura Jiménez', César Cambronero Molina²,
} Diego Moya Castro ${ }^{3}$, Luis Ledezma Cordero 4 \& Santiago Loiza ${ }^{5}$

\begin{abstract}
1. Académico de la Escuela de Ciencias de la Educación (ECE), Universidad Estatal a Distancia (UNED); achinchillaj@uned.ac.cr
2.Profesor de secundaria del sector privado; cescm17@gmail.com

3. Profesor en Sede Occidente, Universidad de Costa Rica (UCR); diegofrancisco.moya@ucr.ac.cr

4. Profesor en Enseñanza de los Estudios Sociales, Ministerio de Educación Pública (MEP); luisantonioledezmacordero@gmail.com
\end{abstract}

5. Investigador independiente; sloaza81@gmail.com

Recibido: 09 de octubre del 2015

Corregido: 12 de abril del 2016

Aceptado: 21 de abril del 2016

\begin{abstract}
Resumen
Esta investigación consta de una revisión sobre la socialización, el capital cultural y social, y la innovación en los procesos educativos; así como de su respectivo trabajo experimental, para analizar y contrastar dichos temas en el contexto educativo costarricense. El objetivo fue analizar los procesos de socialización, de capital social y cultural que poseen estudiantes innovadores de escuelas y colegios, durante el segundo ciclo del año 2014 y el primero del año 2015. Se realizó una investigación de tipo cualitativo mediante el uso de historias de vida. Se encontró que el estudiante innovador proviene de familias en donde el desarrollo del espacio intelectual es muy incentivado. También que sus familiares son muy influyentes y se definen como principales mentores de los entrevistados. Se caracterizó el capital social, y se distinguieron capitales sociales densos e influyentes y capitales de menor tamaño, pero sólidos y de calidad o intensidad. Se determinaron las fuentes de capital cultural. Se encontró gran variedad de elementos tales como la escuela, la familia, actividades sociales, artísticas, recreativas, hábitos cotidianos, libros, entre otros. Se evidenció la ausencia de una metodología específica en el sistema educativo que promueva las innovaciones, labor que recae en los docentes. De este punto sobresale la principal conclusión acerca de la carencia en el sistema educativo costarricense de identificar, comprender y explicar los procesos y las prácticas innovadoras.
\end{abstract}

Palabras clave: educación costarricense, innovación, socialización, capital social, capital cultural.

\section{Abstract \\ Socialization and accumulative processes of social and cultural capital of innovative students in Costa Rican schools and high schools}

This research contains a review about socialization, cultural and social capital, and innovation in educational processes, as well as its respective experimental work in order to analyze and contrast those topics in the Costa Rican educational context. The objective was the analyze of the socialization, and social and cultural capital processes that innovative students have in schools and high schools during the second cycle in 2014 and the first cycle of 2015. A qualitative research was made using life story technique. It was found that innovative students have families where the development of intellectual space is much incentivized. However, theirs family members are very influential being main mentors 
of them. The social capital was characterized, distinguishing thick and influential social capitals and smaller but with more quality social capitals. Cultural capital sources were determined. It was found a wide variety of these sources such as the school, the family, the social, artistic and recreational activities, daily habits, books and others. The lack of a specific methodology in the educational system to promote innovations was seen, such a way it falls in the teachers. Due this, it stands out the main conclusion about de deficiency in the Costa Rican educational system to identify, understand and explain the innovative practices and processes.

Key words: Costa Rican education, innovation, socialization, social capital, cultural capital.

\section{INTRODUCCIÓN}

La sociedad no debe dejar de impulsar y desarrollar dentro del sistema educativo nacional, procesos de enseñanza y aprendizaje que fomenten la innovación. Si la educación no es innovadora es una educación de baja calidad, de tal manera que produce una cultura pobre, visiones cortoplacistas, carencia de deseos de superación y falta de pensamiento crítico.

La escasez de innovaciones en los procesos educativos formales, no solo es producto de la ausencia de herramientas tecnológicas, sino también de la inexistencia de sujetos que promuevan el pensamiento crítico, lateral, abierto e independiente, en contraposición a los enfoques y métodos tradicionales o monótonos. La educación actual, deber ser contextualizada e innovadora con una visión dinámica y una concepción desde la complejidad.

El sistema educativo formal es un agente socializador vital, pues en dichos espacios los estudiantes pueden aprender a comprender, sentir y asumir la vida de forma equilibrada, coherente, ordenada y solidaria; por consiguiente se constituye la educación como un tipo de máquina social de producción y reproducción de personalidades, individuales y colectivas, con formas de vida socialmente aprendidas, naturalizadas, interiorizadas y exteriorizadas (Taberner, 2005).

La socialización se define como:

Un conjunto de experiencias que tienen lugar a lo largo de la vida del individuo y que le permite desarrollar su potencial humano y aprender las pautas culturales de la sociedad en la que va a vivir... Es un proceso que reproduce porque existen interacciones sociales, que permiten que se trasmitan los conocimiento de generación a generación (Macionis y Plumer, 2001, p. 132).

En la vida cotidiana se produce en procesos de socialización y resocialización, igualmente en los espacios educativos formales. Estos procesos de socialización y resocialización fuera y dentro del aula son vitales para entender el desarrollo que tienen las prácticas innovadoras en los centros educativos, debido a que es el contexto donde se propician las condiciones, entre los docentes, los estudiantes y los padres de familia, en cuanto a expectativas, deseos, normas, valores, recompensas y gratificaciones que contribuyen a impulsar dichas prácticas (Martín, 2003, p.39).

Un elemento central en el desarrollo del estudiantado, es el contexto sociocultural familiar y comunal de donde provenga, ya que, el espacio social en donde está inmersa la persona dependiendo del capital 
social y cultural que posea, potencializa o limita su desarrollo personal, social y sus prácticas innovadoras. En los espacios sociales informales, la persona también desarrolla otros capitales sociales y culturales, por ejemplo, en espacios de lectura, de actividades sociales, recreativas, culturales, entre otras; pero, la familia se entiende como el espacio social más cercano y a lo mejor más importante en el desarrollo del individuo, particularmente, durante su infancia y como parte de su proceso de formación identitaria.

En cuanto al estudiante innovador, el desarrollo de su proceso de socialización en el campo educativo puede incrementarse por medio de una educación que propicie una adecuada relación entre la escuela y la familia, y a su vez por el aporte del sistema educativo en el desarrollo de una cultura innovadora. Para José Alois Shumpeter, citado por Avendaño (2012) "la innovación se encuentra estrechamente vinculada con la idea del sujeto innovador, es decir, con aquel que transforma las ideas en inventos, prácticas y acciones... demarcando territorios entre los inventos (una mera idea nueva) y las innovaciones (la introducción exitosa de dicha idea)" (p.189).

Al estudiante que innova, habría que entenderlo como agentes que realizan acciones y prácticas sociales, que implica un registro reflexivo con un significado que pondera la conducta tanto del individuo, como la colectiva (Giddens 2006). Por lo que debe existir la interacción enfocada a identificar, promover y poner en práctica la innovación, pero para que esta exista, primero debe estar presente en los imaginarios de todos estos actores educativos.

Asimismo, los imaginarios

...se constituyen y legitiman a partir de las coincidencias valorativas de las personas, y se manifiestan en lo simbólico a través del lenguaje y en el accionar concreto entre las personas. El imaginario comienza a actuar como tal, inmediatamente que adquiere independencia de las voluntades individuales, aunque necesita de ellas para materializarse (Gamboa, 2011, p. 8).

Por otra parte, una característica intrínseca en la innovación es la creatividad, pues el innovador debe ser a la vez creativo. De hecho, la capacidad de generar ideas y de realizarlas se atribuyen por lo general a la creatividad de la persona (Usua, 2009).

La innovación es una construcción social que da lugar a un espacio donde emergen los capitales sociales y culturales. Tanto el capital cultural como el social, en calidad de promotores de los procesos innovadores, son producto de la interacción individuo-grupo y del intercambio de conocimientos. De la misma forma, los conocimientos y las actitudes hacia la innovación que posee un docente, estudiante o padre de familia, son producto del capital social y cultural acumulado a lo largo de la vida.

Según Jakubowicz (2011), el capital cultural representa las formas objetivadas de la producción cultural, el depósito de los valores culturales construido dentro de un grupo etnocultural con el tiempo, circulado y trasmitido intergeneracionalmente mediante socialización y educación. Este incluye el conocimiento social y los instrumentos para mantener y comunicar el conocimiento.

Para mencionar algunas de las fuentes de capital cultural se pueden citar lo que Fonte y Ranaboldo (2008), llaman "fuentes de identidad territorial" que son diversas: la geografía, la ecología, la identidad étnica, la historia, la arquitectura, las tradiciones y fiestas religiosas, etc. Además, en un estudio sobre el currículo enseñado y el currículo editado, Di Franco, Siderac y Di Franco (2006) se ubica a los libros de textos como portadores y distribuidores del capital cultural.

Bernhardt (2013) considera que "para entender mejor las implicaciones del capital cultural con un contexto educativo, es útil específicamente considerar la concepción del capital cultural en su forma incorporada" (p. 209). Se sabe que una temprana socialización cultural proveída por los padres deja su marca, de manera significativa, durante el resto de la vida del individuo (Kraaykamp y Van Eijck, 2010, p. 210). 
En cuanto al capital social, este es un tejido o red social que soporta e impulsa a la persona o grupo a buscar alcanzar los ideales, objetivos y metas que se proponen obtener en todos los ámbitos de la sociedad. Entre más sólido, amplio, acompañador y retador sea el capital social que se posea, más amplios y retadores serán los objetivos y las metas que se tendrán. Según Bourdieu, citado por Martínez (s.f.) el capital social es el agregado de los recursos actuales o potenciales de que se disponen por pertenecer a un grupo, por la red social más o menos institucionalizada de que se disfrute. En cambio, para Coleman, citado por Ramírez (2005), "el capital social es definido por su función. No es una entidad única, sino una variedad de diferentes entidades... -que- facilitan la realización de ciertas acciones para los actores dentro de la estructura" (p. 26). Por otra parte, Putnam citado por Ramírez (2005), se centra en el aporte de elementos nuevos, por ejemplo, la confianza, que es esencial para fortalecer las relaciones sociales, pues permite el aporte a otros tipos de capital.

Partiendo del concepto de capital social, el papel de un innovador siempre es producto de un componente social, principalmente en la escuela y en el aula donde se desarrolla, no solo son espacios donde el niño o adolescente crece adquiriendo conocimientos, sino que son espacios socializadores, donde las redes entre los estudiantes permiten todo tipo de intercambios, y existe lugar para el desarrollo de ideas innovadoras.

Considerando estos planteamientos, es acertado decir que las investigaciones acerca de las prácticas educativas innovadoras en los procesos educativos formales costarricenses, a la luz de un enfoque socioeducativo y sociocultural, poseen actualidad, pertinencia, importancia y urgencia innegables para el país. El objetivo del proceso investigativo que sustenta este artículo, fue analizar los procesos de socialización, de capital social y cultural que poseen los estudiantes innovadores de escuelas y colegios Atención Prioritaria los Jardines de San Ramón, Primo Vargas Valverde de Orotina, Colegio Bilingüe de Palmares, Escuela de la Unión y Escuela de San Francisco de San José, durante el segundo ciclo del año 2014 y el primero del año 2015.

\section{MATERIALES Y MÉTODOS}

\section{Tipo de investigación}

La investigación se sustentó en el paradigma epistemológico y metodológico naturalista. El enfoque fue de tipo cualitativo, con carácter descriptivo, exploratorio, analítico y prospectivo. Se realizó una investigación transversal es decir, se aplicó el trabajo de campo y se investigaron las innovaciones en un único y específico momento, pero dado que el estudio reconstruye la historia de vida de los innovadores, también tuvo un alcance temporal longitudinal retrospectivo.

Respecto a la triangulación, se contrastó lo que dice la teoría sobre las innovaciones, la socialización, los capitales social y cultural, con los datos y la información obtenida en el trabajo de campo realizado. En la historia de vida se platearon varias preguntas cuyo fin era reconfirmar, validar, y triangular lo que dijo el estudiantado participante.

\section{Sujetos de investigación}

El trabajo de campo se realizó con estudiantes innovadores de los siguientes centros educativos: Colegio Bilingüe de Palmares; Escuela Atención Prioritaria Los Jardines, San Ramón; Escuela Primo Vargas Valverde, Orotina; Escuela de la Unión, Cartago y Escuela de San Francisco, San José. Para seleccionar 
los entrevistados, se tomó como base una investigación elaborada por sociólogos de la Universidad Nacional, de donde se extrajo las características que suelen tener las personas innovadoras. Se buscó en las escuelas y colegios, donde dieron permiso para realizar el trabajo de campo, estudiantes que tuvieran varias de estas características, sobre todo las relacionadas con la disposición al cambio, el pensamiento lateral, la actitud y las prácticas frecuentes de estar averiguando, preguntando, investigando sobre cuestiones novedosas y la pasión y vocación por estar haciendo los proyectos, tareas e investigaciones de modo diferente y mejor, entre otras.

\section{Recolección y análisis de la información}

Se utilizaron dos técnicas de corte cualitativo: la historia de vida (anexo 2) y el análisis de contenido de carácter cualitativo.

Se interrogó al estudiantado innovador sobre diferentes aspectos personales, académicos y familiares mediante una serie de preguntas previamente estructuradas con el fin de reconstruir sus historias de vida a partir de lo verbalizado. La información recabada, producto de las ocho historias de vida, se transcribió literalmente. Luego se construyeron categorías de análisis que permitieron agrupar, a partir de criterios de inclusión y exclusión establecidos, las ideas, opiniones, valoraciones y expectativas, parecidas o diferentes, precisas o ambiguas. La construcción y ordenamiento de categorías de análisis, se realizó mediante la elaboración de matrices con columnas y filas, donde se incluyeron literalmente todas las respuestas de los sujetos consultados, según una determinada pregunta o tema. Una vez elaborada la matriz, se procedió a leer y valorar cada respuesta, poniéndole atención a lo que dijo cada uno, pero también a lo dicho en general. Posteriormente, se elaboró una lista de criterios analíticos y de toda la información contenida en las matrices, se seleccionaron párrafos literales que contenían los resultados más relevantes. Finalmente, se hizo el respectivo análisis y la interpretación de los resultados, tomando en cuenta la teoría de los temas estudiados.

\section{RESULTADOS}

\section{Caracterización de la familia de los estudiantes innovadores}

La mayoría de los entrevistados no provenían de familias de un estrato económico elevado. En general dijeron tener familias inquietas, conversadores, que leen periódicos o libros, realizan actividades sociales y debates sobre diversos temas. Todos los entrevistados tienen familias que se interesan de forma frecuente o tienen disposición por utilizar nuevas tecnologías. De manera que el desarrollo del espacio intelectual es muy incentivado.

Sobre el tema respecto a cómo fue la infancia, si hubo algo particular o diferente, si tuvo muchos compañeros desde muy pequeño, si los padres les leían cuentos, novelas, fábulas, y otros, la mayoría de los entrevistados afirman tener una infancia feliz a pesar de ciertas adversidades afrontadas. 


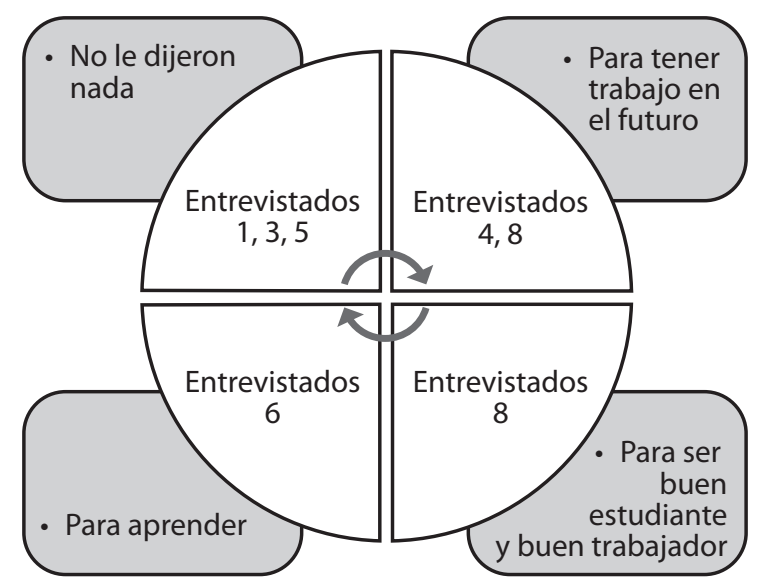

Figura 1. Por qué razones los padres les dicen a los estudiantes innovadores que deben estudiar. Fuente: elaboración propia.

\section{Influencia de la familia en el capital social de los entrevistados}

Al preguntarles acerca de qué les decían sus padres y madres sobre la educación, la totalidad del estudiantado participante mencionó que para su familia la educación es muy importante para la vida. En relación con si en la educación hay que destacar y ser diferente, cuatro de los entrevistados dijeron que sí debe ser así. Respecto a hacer las cosas diferentes y mejores, la mitad mencionaron que siempre les decían que debían hacerlas de esa forma.

Se externaron otros aspectos vinculados sobre lo que les decían su padre y madre sobre la educación. Se profundizó en las razones por las cuales les decían esas cosas (figura 1). Acerca de la expectativa parental se evidenció de forma clara e importante en relación al futuro laboral de sus hijos e hijas.

\section{La influencia de la familia de los entrevistados en las prácticas innovadoras}

Se les consultó al estudiantado innovador que fue lo que le dijeron el papá y la mamá que los hizo empeñarse en hacer los trabajos y los proyectos educativos diferentes y mejores, si lo recordaban, qué fue lo que les dijeron, cómo y por qué eso los cambió (figura 2). A modo de síntesis, un estudiante señaló: "Mis padres me dijeron que tenía que trabajar bien y mejor en la escuela, porque si no, no podría ser alguien importante en la vida. Yo creo que todo lo que yo haga mejor en el colegio, me ayudará a ser una persona mejor...".

Se indagó si los padres y madres los recompensaban cuando hacían los trabajos y los proyectos educativos diferentes y mejores, se determinó que la mayoría de estudiantes innovadores consultados reciben recompensas, ya sea motivación verbal, obsequios, viajes, comidas especiales o permisos para asistir a ciertas actividades. 


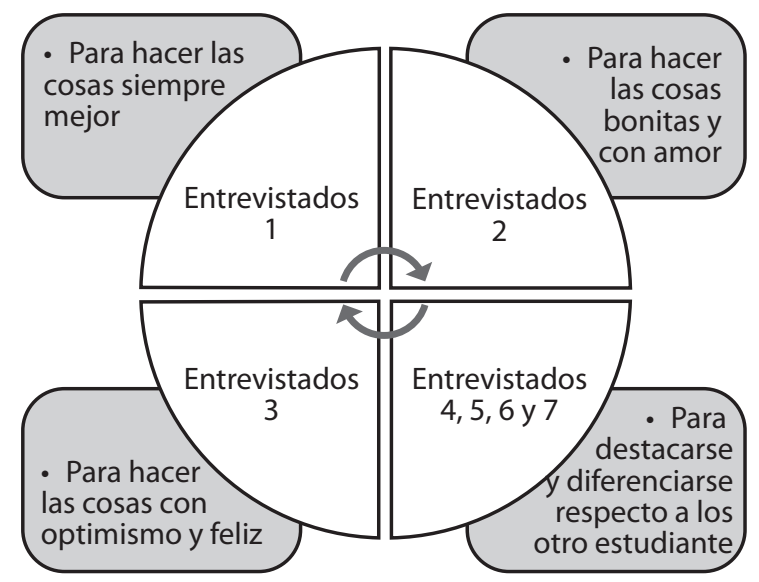

Figura 2. Lo que los padres le dijeron a los estudiantes para que realicen trabajos novedosos en el centro educativo. Fuente: elaboración propia.

\section{La influencia en los estudiantes innovadores de otros actores sociales}

Se determinó cuáles fueron las personas que conocieron que tuvieron más influencia sobre la forma de actuar diferente y mejor (figura 3), por lo tanto se encontraron actores a nivel familiar, dentro y fuera del hogar, a nivel escolar y extrafamiliar; así como personas modelos o mentores. Se descubrió que en todos los casos tienen a sus padres y madres como principales mentores, o por lo menos forman parte importante de su capital social. Solamente dos entrevistados señalaron otras dos figuras adicionales fuera del núcleo familiar como mentores.

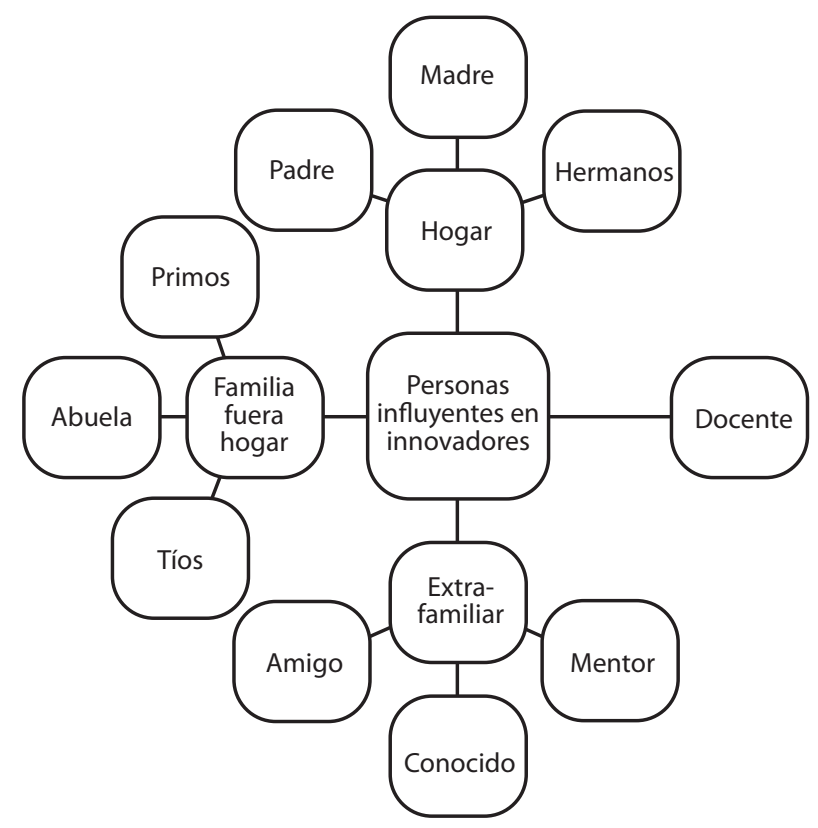

Figura 3. Influencia de actores sociales en entrevistados. Fuente: elaboración propia. 


\section{Caracterización del capital social en estudiantes innovadores}

Se encontraron diferencias, en cuanto al tamaño del capital social de cada estudiante. En un entrevistado (figura 4), se encontró que el tamaño del capital social, medido por la cantidad de amigos y compañeros que tuvo, fue reducido, debido a la poca relación que mantuvo con ellos en la escuela y el colegio. Un aspecto similar está presente en otro de los entrevistados, quien también evidenció escasa pertenencia hacia grupos sociales, pero sí mostró un aporte importante por la interacción con su familia y con sus maestros (figura 4). El capital social de los otros entrevistados se encontró aún más denso, pues ellos establecieron mayor número de relaciones beneficiosas, dentro de los diferentes grupos.

El capital social se puede ver afectado por la individualidad o la colectividad del estudiantado innovador. Se observó capacidad y decisión individual en tres entrevistados, dado que mencionaron que realizan los trabajos educativos y se desempeñan en la escuela y el colegio de forma diferente, producto de las decisiones personales y motivaciones propias, sin que medie tanto la influencia de otras personas, pero sin rechazarla del todo. Cuatro de los entrevistados, mostraron lo contrario, pues experimentaron una mayor participación y aporte de las colectividades en sus vidas, que evidencia la presencia de un capital social más amplio e influyente en su papel de estudiantes innovadores, como lo explica el entrevistado 2 (figura 4).

El capital social es un proceso interactivo entre las partes, basado en reglas y normas que establecen desde un principio los que forman el grupo social. Esto se identificó mayormente en tres de los

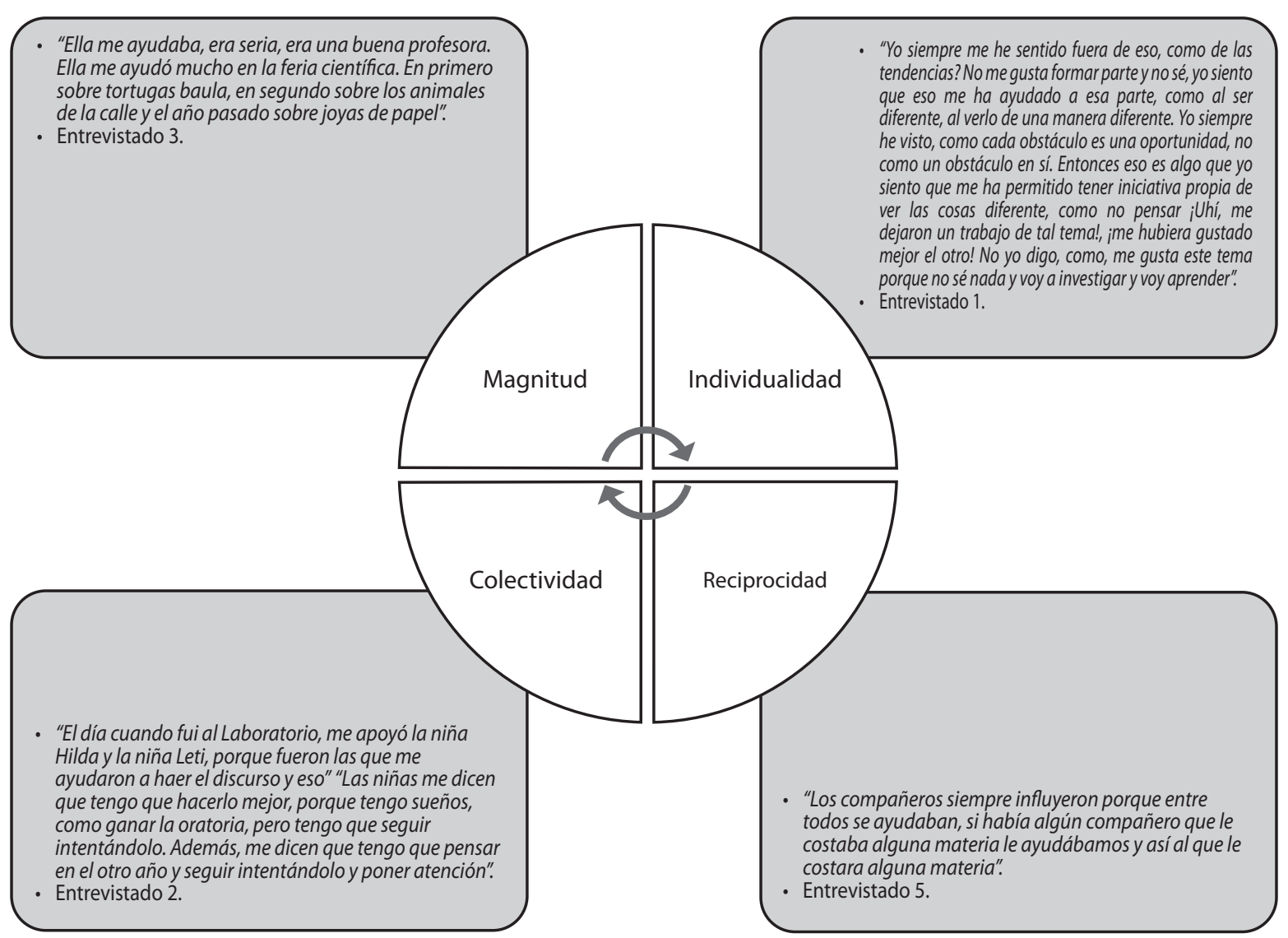

Figura 4. Caracterización del capital cultural de los estudiantes innovadores. Fuente: elaboración propia. 
entrevistados, los cuales mencionaron un mayor aporte en el capital social de las demás personas hacia ellos, principalmente de compañeros de escuela o colegio y viceversa, por lo cual se dio una mayor interacción de aprendizajes y experiencias, es decir reciprocidad (figura 4).

\section{El capital cultural del estudiantado innovador}

Se determinó la influencia que tiene el capital cultural en la innovación que realizan los entrevistados y se indagó respecto a las fuentes de adquisición de este durante su infancia. En general, se encontró que todos gustan de usar nuevas tecnologías, es decir, todos aquellos últimos desarrollos tecnológicos y a sus respectivas aplicaciones, como el uso de teléfonos celulares, leen periódicos, libros o ven las noticias. Solamente el entrevistado 1, detalló aspectos más que todo de la parte afectiva de su familia (figura 5). Tres de los entrevistados destacaron que su familia realiza alguna actividad social como ir a bingos o eventos en la comunidad. Durante su infancia, en dos estudiantes, se vio que no viajaban mucho, los demás, si realizaban paseos o visitaban a sus familiares (figura 6).

En todos los entrevistados, se encontró que los familiares les leían cuentos, o en su lugar les contaban historias y anécdotas de sus antepasados. Como lo describe uno de los sujetos: "Mi papá a veces me contaba de la infancia de él, me contaba que ellos vivían juntos en una pequeña cabañita. Él tuvo que dejar la escuela para ayudar a mi abuelo a chapear con un cuchillo en el monte, me contó que él hacía sus propios juguetes, que ellos dormían en unos sacos y todo eso".

Sobre otras fuentes de capital cultural se les consultó sobre la frecuencia en asistir a bibliotecas, librerías, cine, festivales musicales y literarios. Hay coincidencia en la mitad de los entrevistados en asistir en ocasiones a la biblioteca, ya sea la del centro educativo, de la localidad o de sus hogares. En cuanto a librerías solo uno mencionó asistir. Además pocos mencionaron haber ido a festivales musicales o literarios. De forma general, todos los estudiantes innovadores van al cine, algunos con más frecuencia que otros (figura 6). Estas actividades las llevan a cabo con sus familiares u otros allegados o amistades.

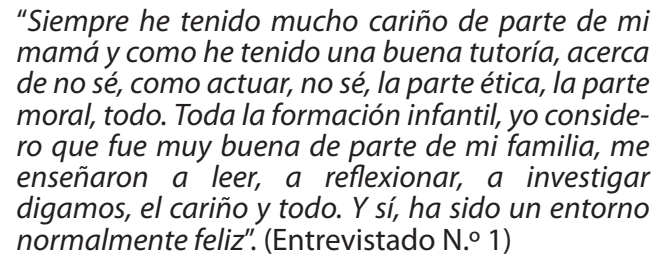
mamá y como he tenido una buena tutoría, acerca de no sé, como actuar, no sé, la parte ética, la parte moral, todo. Toda la formación infantil, yo considero que fue muy buena de parte de mi familia, me enseñaron a leer, a reflexionar, a investigar digamos, el cariño y todo. Y sí, ha sido un entorno normalmente feliz". (Entrevistado N. ${ }^{\circ}$ 1)

\begin{tabular}{|c|c|}
\hline $\begin{array}{l}\text { ELC } \\
\text { CULTU } \\
\text { ESTU } \\
\text { INNOI }\end{array}$ & $\begin{array}{l}\text { ITAL } \\
\text { DE LOS } \\
\text { NTES } \\
\text { ORES }\end{array}$ \\
\hline $\begin{array}{l}\text { “... tenemos televisores, computadora, una peque- } \\
\text { ña de mi mamá... sobre las tecnologías, si las } \\
\text { usamos mucho, tenemos todo ese tipo de cosas } \\
\text { (teléfono, celular), tengo un DS y un reproductor de } \\
\text { música y nada más". (Entrevistado } \mathrm{N} .^{\circ} \text { 6) }\end{array}$ & $\begin{array}{l}\text { "Yo leo mucho, también veo programas eductati- } \\
\text { vos. Todo eso me ayuda a mejora lo que hago con el } \\
\text { trabajo en la escuela". (Entrevistado N. } 3 \text { ) }\end{array}$ \\
\hline
\end{tabular}

Figura 5. El capital cultural de los estudiantes innovadores. Fuente: elaboración propia. 


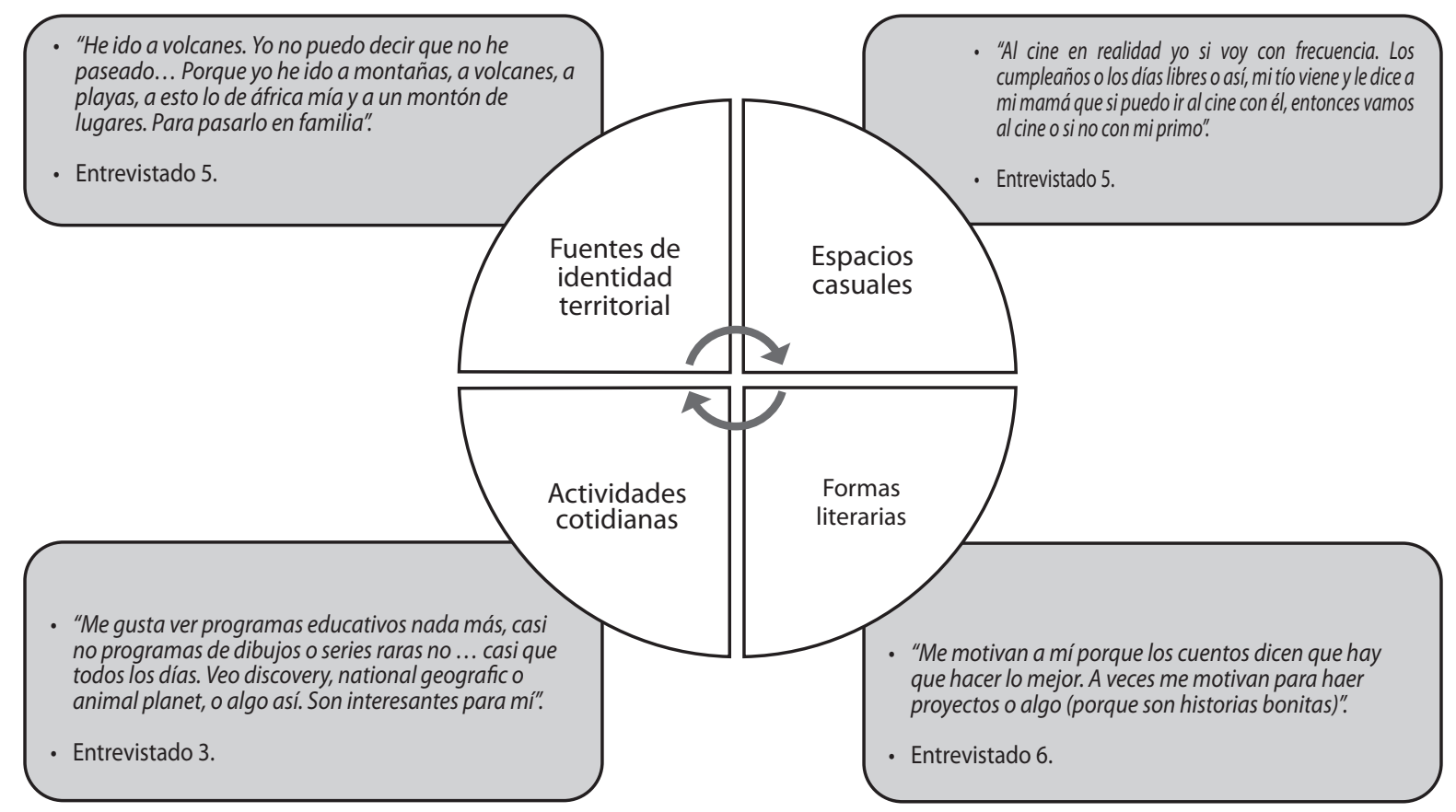

Figura 6. Fuentes de capital cultural. Fuente: elaboración propia.

Refiriéndose a otras actividades que realizan los entrevistados y sus familiares, se observó que la mayoría participa en alguna actividad artística o cultural, o que han asistido a algún museo, evento social o deportivo.

Respecto a la costumbre de ver televisión, se encontró que cuatro de los entrevistados prefieren ver programas de entretenimiento, ya sea infantiles, deportes, series o novelas. Su frecuencia es variada. Uno de los entrevistados difiere, ya que opta por ver programas educativos (figura 6). En dos casos hubo una noticia, investigación u otra cosa que los motivó cambiar la forma de realizar los trabajos y los proyectos educativos. Por ejemplo, un entrevistado comentó: "Sí, creo que fue de un muchacho que no podía escribir muy bien porque le faltaba un brazo, entonces fue eso, un reportaje".

En cuanto a la educación formal (escuela) y la educación brindada por la familia, como proveedores de capital cultural, es importante destacar que en la mitad de los entrevistados, se presentó un hecho que refuerza la adquisición de capital cultural y la innovación. En contraste, en el caso de uno de los estudiantes fue proporcionado por la madre y en otro fue inculcado por la escuela (figura 7).

Sobre la preferencia por la lectura, la mayoría de los sujetos gustan de leer libros de historias o cuentos. Solamente uno prefiere leer libros de carácter científico. Algunos coinciden en que les ayuda a aprender a leer y desarrollar su vocabulario, además de la distracción, diversión o motivación que le genere el libro o la historia y por el aprendizaje extra que obtienen. 


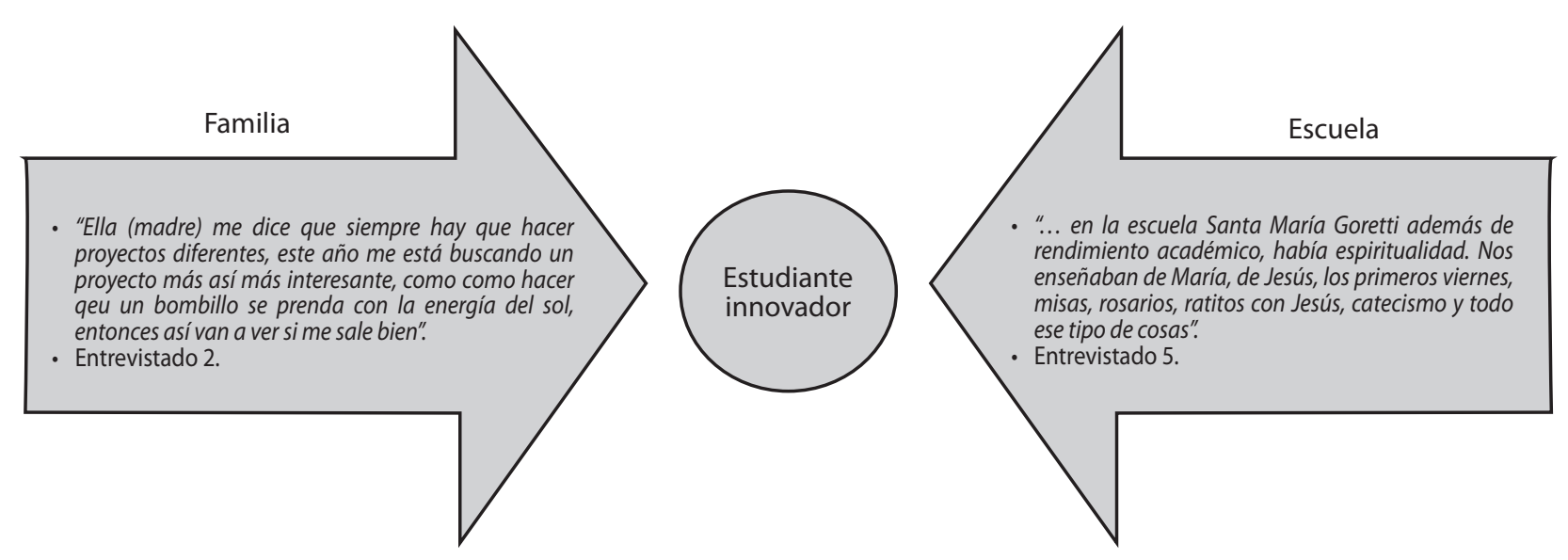

Figura 7. Proveedores de capital cultural de los estudiantes innovadores. Fuente: elaboración propia.

\section{La influencia del sistema educativo en el estudiantado innovador}

Una de las tareas básicas del sistema educativo debe ser desarrollar en los estudiantes la capacidad de innovación. Sin embargo, se encontró que se está muy lejos de cumplir. Ante la pregunta ¿Cuándo llegó al centro educativo, escuchó algo que lo motivó a hacer los trabajos y los proyectos educativos de manera diferente y mejor?, cinco de ocho estudiantes contestaron con un "No". Esta respuesta, aunque simple y corta, no deja de ser alarmante, pues proviene de más de la mitad de los estudiantes y muestra la falta de acción por parte del sistema educativo en el tema de la innovación. Además, se pudo observar, que un entrevistado nunca mencionó a la escuela como importante o influyente. Este vacío, se ve relativamente compensado por los profesores. Ante la pregunta, ¿le estimula el docente para que haga los trabajos y los proyectos educativos de forma diferente y mejor?, uno de los entrevistados respondió: "El profe Erik, la niña Xinia y la niña Hilda, me han motivado a hacer los trabajos y los proyectos educativos de manera diferente y mejor... Los docentes me decían, Fiorela usted tiene que estudiar, porque cuando usted esté en el segundo año, va a ver las mismas cosas, pero diferente. La niña Xinia me decía, tiene que ser mejor, tiene que aprender...". Este tipo de respuesta fue la norma para seis de los ocho entrevistados, lo cual deja una pista del gran rol que tiene el profesorado.

\section{DISCUSIÓN DE RESULTADOS}

La familia tiene una gran influencia en el desarrollo de niños y niñas innovadores, ya que la totalidad de ellos dijeron estar convencidos de que la educación es vital para su desarrollo humano y laboral, y tales concepciones fueron inculcadas por sus padres. El capital social que ha construido el estudiantado innovador con sus padres y madres, los motiva e impulsa a desarrollar innovaciones en sus instituciones educativas. Visto así, los entrevistados quieren ser un ejemplo en el tema de las innovaciones y con esto labrarse un buen futuro y responder a las expectativas de sus padres. De tal manera que la innovación en el sistema educativo se convierte en un medio que les permitirá alcanzar el éxito futuro, y el incentivo que reciben de los familiares, en un agente motivador. 
Es claro que la motivación, los estímulos y las frases positivas que le trasmite la familia al estudiantado lo estimulan y de cierto modo lo retan a realizar los trabajos educativos de mejor manera. Como parte de las motivaciones recibidas por los entrevistados, se destacan las recompensas de origen parental, familiar o docente, cuando hacen los trabajos y los proyectos educativos diferentes y mejores, lo cual muestra la importancia que tiene el incentivo en el contexto del capital social en la formación de sujetos innovadores. Desde el punto de vista del capital cultural, las recompensas materiales, pasan a convertirse en formas objetivadas de capital cultural que en ocasiones pueden ser muy valorizadas por los sujetos.

Las personas del hogar fueron las más influyentes en el estudiantado innovador. Pero la familia que va más allá del hogar paterno, también influyó en algunos de los entrevistados para empezar a hacer los trabajos y los proyectos educativos de forma diferente y mejor. Un grupo importante de los sujetos tuvo buenos ejemplos fuera del hogar paterno, los cuales se distinguieron por ser especialmente buenos motivadores.

Respecto a los modelos o ejemplos que tuvieron los entrevistados, estos se distinguieron por ser personas que realizan sus actividades buscando la manera de cambiarlas y mejorarlas, por lo que justamente son considerados como referentes importantes para la vida del estudiantado. Esta persona o modelo formará parte del capital social y se podría convertir en un punto de referencia o en un ejemplo por imitar; es decir, pasará a ser un mentor, el cual siempre estará presente en el imaginario del innovador, de hecho, formará parte de su vida, dado la relevancia e incidencia que tiene sobre él.

Según esta investigación, en algunos casos, los padres y madres de familia fueron los principales mentores. También un determinante, dentro del capital social de estos estudiantes es el maestro, puesto puede observar, dirigir y conducir, más de cerca el desempeño de un estudiante innovador dentro del proceso de enseñanza-aprendizaje.

El estudiantado requiere para poder materializar sus innovaciones, sentirse retado por un docente exigente y también de cierta manera innovador. Se debe tener presente que este tipo de estudiantes suelen ser más innovadores cuando están insertos en escenarios donde lo emocional, el reto, la necesidad, las expectativas y los socioemocionales también inciden sobre las innovaciones.

En cuanto a la caracterización del capital social del estudiantado innovador, el tamaño dependerá en gran medida de la disponibilidad social que el innovador pueda utilizar objetivamente. Por lo que es posible que algunos estén dando sus primeros pasos en el proceso de construcción de su propio capital social, dentro del cual se ven envueltas gran cantidad de relaciones, que irán enriqueciendo la magnitud y densidad del capital social en un proceso de confianza y reciprocidad de conocimientos. Sin embargo, existe un aspecto importante que podría darse en otro tipo de estudiantes: mantener relaciones más estables y estructuradas.

La magnitud y la incidencia sobre la innovación del capital social de algunos de estos entrevistados, se debe prioritariamente al aporte de sus familias, pues es en esta donde comienzan a desarrollar y afianzar sus relaciones interpersonales y sus deseos de innovar.

Sobre la colectividad o individualidad del capital social, según lo encontrado, un estudiante puede convertirse en innovador sin necesidad de tener una gran cantidad de relaciones con diferentes grupos, ante lo cual se podría decir que las innovaciones se deben mayoritariamente a causas individuales, aunque siempre exista influencia de otros actores sociales.

En caso contrario, se observó en otros sujetos que la innovación se desarrolló en buena medida por las relaciones e influencias que recibieron los estudiantes innovadores por parte de otras personas; tanto así que en todo momento señalan y destacan los aportes de los docentes, familiares y hasta amigos. 
En lo que respecta a la reciprocidad del capital social, se demuestra que para establecer bases sólidas en el capital social de un estudiante innovador, las mismas deben de forjarse produciendo una amplia reciprocidad entre los grupos sociales determinados, tanto de quien aporta dentro de la red establecida, como también del que asume la obligación que implica la confianza que todo grupo social requiere para reproducirse.

En algunos estudiantes entrevistados, su papel de innovadores fue más desarrollado por la relación de confianza y trabajo colaborativo que establecieron todos los integrantes del grupo.

Según esta investigación, las dos principales fuentes de capital social y cultural en estudiantes innovadores, lo constituyen la familia y la escuela. La escuela es sin lugar a duda vital para el estudiante innovador, por el aprendizaje cognitivo, actitudinal y conductual que este espacio les brinda. Por su lado, los padres y madres de familia son los primeros y esenciales responsables respecto a la cantidad y calidad del capital social que posee el estudiante.

En términos de capital cultural, en algunos de los entrevistados, sobresale la enseñanza de la madre y su participación en las tareas escolares de su hijo o hija. Al decirle que siempre hay que hacer cosas diferentes, lo está impulsando a innovar, le está dando el ejemplo, le muestra el interés de ella por la educación de él. En cuanto al papel del centro educativo, se notó en otros entrevistados, que recibieron una enseñanza distinta, ya sea por la solicitud de elaborar materiales o tareas diferentes, es decir poner en práctica diversas metodologías o por inculcar valores y creencias más allá de lo académico. En este sentido, la escuela de acuerdo con las estrategias pedagógicas y ambiente educativo que desarrolle, puede también ser un agente innovador muy importante.

Acerca de la influencia que tiene el capital cultural en la innovación que realizan los entrevistados y sobre las fuentes de adquisición de este durante su infancia, se encontró una considerable importancia dada a la lectura de los textos y al uso de nuevas tecnologías, tanto en ellos como sus familiares. Además, retomando lo que Kraaykamp y Van Eijck (2010) afirman, es claro que "un clima de casa intelectual favorece el desarrollo del gusto por la cultura [De manera que] los padres culturalmente activos incrementan el capital cultural incorporado de sus hijos, al funcionar como un ejemplo directo para los niños" (p. 213).

Sobre los lugares que visitaron, conocieron o donde han vivido, constituyen, como lo definen Fonte y Ranaboldo (2008), fuentes de identidad territorial específica que forman parte de la cultura o del capital cultural, sobresaliendo aspectos de los cuales las personas pueden aprender, ampliando su capital cultural, favoreciendo la actitud innovadora.

Otra de las fuentes de capital cultural fue constituida por las historias, cuentos o canciones empleados por sus familiares, las cuales son formas de capital cultural que pudieron servir de enseñanza o motivación a los estudiantes innovadores. Además, pueden permitirles llegar a ser más inteligentes e innovar, al ser formas literarias o verbales que usan las palabras para transmitir la información o conocimiento (Sullivan, 2001, citado por Wang, 2012).

En lo que respecta a otras fuentes, tales como asistir a bibliotecas o librerías, todos los estudiantes entrevistados mencionaron haber ido y todavía tener una gran curiosidad por la lectura. Algunos de ellos, incluso participaron o asistieron a festivales literarios y musicales, lo cual refleja un gusto por la cultura. Todas estas actividades cotidianas o casuales las llevaron a cabo con sus familiares, allegados y amistades, de cuya interacción puede provenir más capital cultural para ellos por socialización e intercambio cultural intergeneracional.

El participar en algún grupo social, artístico o deportivo, les permite socializar, intercambiar ideas, adquirir nuevas destrezas, poner en práctica sus habilidades propias, desarrollo personal y gusto por la cultura. 
Respecto a la costumbre de ver televisión, esta constituye una fuente de capital cultural que además de llamarles la atención o distraerlos, es un elemento importante de socialización y globalización que influye directamente en la manera de ver el mundo, pensar y actuar de los sujetos. Visto así, cualquier aspecto innovador observado en la televisión por el estudiantado innovador, puede motivarlos y provocar un cambio en sus imaginarios, que les puede servir de ejemplo para realizar innovaciones, como ocurrió en dos casos, en los cuales hubo una motivación obtenida por una noticia o investigación.

Otro aspecto fundamental por considerar, es el papel de los libros de texto, libros en general y de la lectura como proveedores de capital cultural y su efecto en la educación e innovación. Como se describió, la mayoría tenía gusto por la lectura. Retomando a Di Franco, Siderac y Di Franco (2006), los libros son portadores y distribuidores del capital cultural y ejercen una fuerte impronta a la hora de construir representaciones identitarias.

Respecto a la influencia del sistema educativo en los estudiantes innovadores, ante la ausencia de una metodología específica promotora de innovaciones, el docente actual no tiene más remedio que cumplir un papel protagónico en la formación de los estudiantes innovadores. De ahí que ante los cambios actuales, el docente también entienda y asuma la tarea de impulsar y regular dichos cambios, a fin de que no se convierta en una figura anacrónica, incapaz de poder identificar, entender y actuar de modo apropiado en la sociedad actual.

Asimismo, es alentador saber que hay profesores que se salen del esquema para ayudar a motivar a los estudiantes. Pero no se puede esperar que los profesores hagan todo el trabajo. La innovación en los procesos educativos es resultado no solo de la interacción que haya entre los estudiantes y los docentes, sino también de las interacciones fuera del espacio educativo con los miembros del hogar y hasta de los grupos de pares.

\section{CONCLUSIONES}

Un contexto familiar adecuado, facilita la posibilidad del surgimiento de niños innovadores. El incentivo por parte los familiares hacia sus hijos, es un agente motivador para el innovador. Sobresalen las altas expectativas educativas de los padres hacia los entrevistados y el uso de recompensas cuando hacen los trabajos y los proyectos educativos diferentes y mejores. La importancia que le dan los padres de los entrevistados a la educación y la influencia sobre ellos en ver la educación como un medio para el éxito laboral, profesional y personal, favorece el deseo de hacer las cosas diferentes y mejores, para lograr potencializar el carácter innovador.

El capital social dependerá en gran medida de la influencia que tienen los padres de familia en el niño o adolescente y de las relaciones que ellos también construyan con otras personas. Pero también del entorno educativo y de los espacios recreativos. En algunos casos, el estudiante puede desarrollarse como innovador por razones individuales y con escasa influencia de otras personas. Sin embargo, a lo largo de la vida el innovador siempre se relaciona con otras personas de las cuales aprende. Los mentores dentro del capital social, son de gran importancia, pues podrían aumentar la motivación y el conocimiento en los niños o adolescentes innovadores, mediante el aporte que tengan hacia estos.

El capital cultural incorporado proporcionado por el entorno y afecto familiar, se convierte en un hecho que lleva al sujeto a la innovación y ver la educación o la vida de una forma diferente. Las fuentes de capital cultural en los estudiantes innovadores son muy variadas. Dentro de las cuales se destacan principalmente el visitar, conocer o vivir en otros lugares, el gusto por la lectura, la asistencia a bibliotecas o librerías, la participación y práctica de actividades artísticas, deportivas o sociales, el ver televisión, entre otras. 
El sistema educativo costarricense carece de identificar, explicar y comprender los procesos y las prácticas innovadoras. Debiendo ser lo contrario puesto que es un espacio rico en capital social, donde se debe dar lugar al desarrollo de ideas innovadoras.

\section{REFERENCIAS}

Armenteros. M, Medina. M, Ballesteros. L y Molina. V. (2012). Las prácticas de gestión de la innovación en las micro, pequeñas y medianas empresas: resultados del estudios de campo en piedras negras Cohauila, México. Revista Internacional de administración y finanzas. Vol, 5 N4, p.p 29-50. Disponible en: http://scholar.google.es/scholar?hl=es\&q=Mar\%C3\%ADa+del+Carmen+Armenteros+Acosta-+pr acticas+innovadoras\&btnG=\&|r=

Avendaño, W. (2012). Innovación: un proceso necesario para las pequeñas y medianas empresas del municipio de San José de Cúcuta Norte de Santander, Colombia. Revista semestre económico. Vol. 5 N. 31, p.p 187-208. Recuperado el 18 de marzo del 2013 en http://dialnet.unirioja.es/servlet/ articulo?codigo $=4008333$

Bernhardt, P. (2013). The advancement via individual determination (AVID) program: Providing cultural capital and college access to low-income students. School Community Journal, vol. 23, N. 1, pp. $203-222$. Disponible en: http://files.eric.ed.gov/fulltext/EJ1004339.pdf

Bourdieu, P. (2005). Capital cultural, escuela y espacio social. Siglo XXI editorial. Buenos Aires, Argentina.

Di Franco, M., Siderac, S. y Di Franco, N. (2006). Del curriculum enseñado al curriculum editado. Praxis Educativa (Arg), N. ${ }^{\circ}$ 10, pp. 81-87. Disponible en: http://www.redalyc.org/pdf/1531/153114357010. pdf

Fonte, M. y Ranaboldo, C. (2008). Desarrollo rural, territorios e identidades culturales: perspectivas desde américa latina y la unión europea. Revista Opera, N. 7, Mayo, pp. 9-31. Disponible en: http://www. redalyc.org/articulo.oa?id $=67500702$

Giddens, A. (2006) La constitución de la sociedad: Bases para la teoría de la estructuración. Amorrurtu editores. Buenos Aires. Argentina.

Jakubowicz, A. (2011). Playing the triangle: cosmopolitanism, cultural capital and social capital as intersecting scholarly discourses about social inclusion and marginalisation in Australian public policy debates. Cosmopolitan Civil Societies Journal, vol. 3, N. 3, pp. 68-91. Disponible en: http://epress.lib.uts.edu. au/journals/index.php/mcs/article/viewFile/2215/2574

Kraaykamp, G. y Van Eijck, K. (2010). The intergenerational reproduction of cultural capital: a threefold perspective. Social Forces, vol. 89 (1), pp. 209-232. Disponible en: http://ics.uda.ub.rug.nl/FILES/root/ Articles/2010/KraaykampG-Intergene/2010-KraaykampG-Intergenerational.pdf

Macionis, J. y Plumer, K. (2001) Sociología. Editorial Prentice-Hall. Madrid, España.

Martínez, J. (s. f.). Las clases sociales y el capital en Pierre Bourdieu: un intento de aclaración. Recuperado el 26 de agosto, 2014 en: http://josamaga.webs.ull.es/Papers/clase-bd-usal.pdf

Matos, J. (2001) El problema de la sociología y el imaginario social en el pensamiento clásico y contemporáneo. Centro Universitario de IMIAS, Guantánamo. 2011

Ramírez, J. (2005). Tres visiones sobre capital social: Bourdieu, Coleman y Putnam. Acta republicana política y sociedad, 4(4), p.p 21-36.

Taberner, J. (2005). Sociología y educación: el sistema educativo en sociedades modernas, funciones, cambios y conflictos. Tecnos, editorial. Madrid.

Usua, N. (2009). De la Información al conocimiento; Del Conocimiento a la Innovación. Madrid, España: Plaza y Valdes.

Wang, H. (2012). The effects of cultural capital on educational aspirations among adolescents in Macau. Chinese Sociological Review, vol. 44, N. ${ }^{\circ}$, pp. 52-75. 
